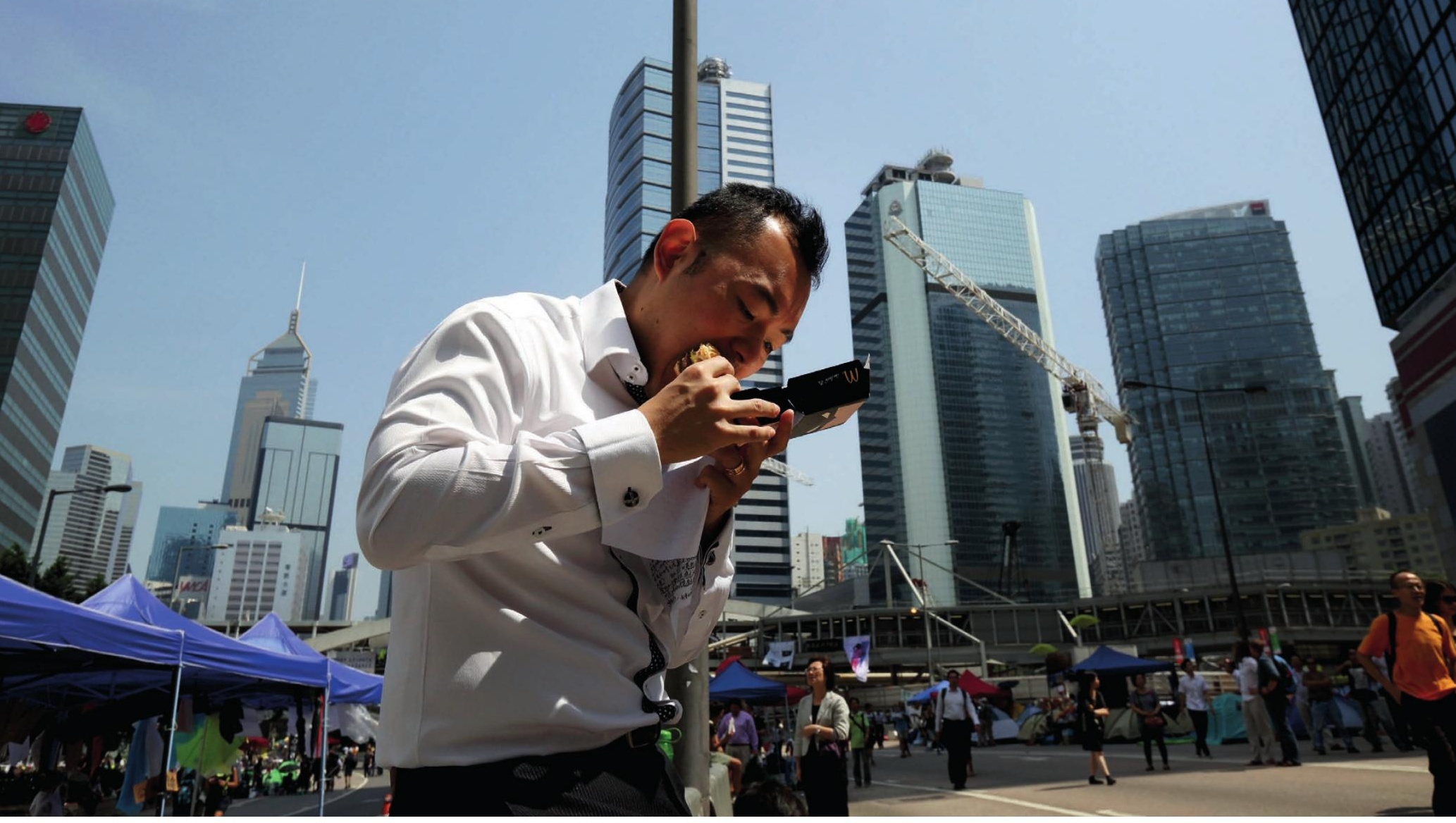

City living and consuming convenience food are two of the factors that researchers think could be behind the increase in inflammatory bowel disease.

\title{
EPIDEMIOLOGY
}

\section{Rising in the East}

\section{Inflammatory bowel disease is a growing problem in Asia. But that increase presents a golden opportunity for research.}

\section{BY KELLY RAE CHI}

$\mathrm{T}$ he gastroenterology ward at the Prince of Wales Hospital in Hong Kong has undergone a dramatic change under the watchful eyes of gastroenterologist Siew $\mathrm{Ng}$ and her colleagues. When Ng started seeing patients there nearly seven years ago, she mainly encountered infectious diseases such as intestinal tuberculosis that ravage the gastrointestinal tract. But today, the ward is overwhelmed by young men and women with inflammatory bowel disease (IBD), a lifelong gastrointestinal condition that can be debilitating if left untreated (see 'Preparing for the burden'). "I can hardly keep up with all the patients I have," says Ng. "The number of cases is basically exploding."

And she has the data to support this statement. Ng also leads the Asia-Pacific Crohn's and Colitis Epidemiology Study (ACCESS), which, for the past five years, has tracked new cases of Crohn's disease and ulcerative colitis - the two major forms of IBD - across 13 countries in Asia as well as Australia. The incidence of IBD in Hong Kong has grown from just 1 case in a million in 1985 to a little
Incidence of IBD per million people

in Hong Kong
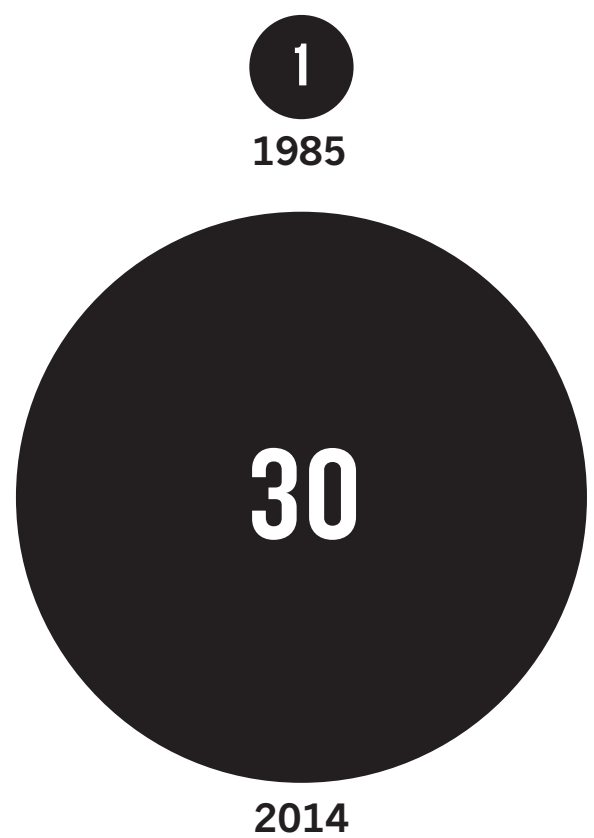

over 30 in every million in 2014 (ref. 1).

In countries such as the United States, the United Kingdom and Canada, the rate of IBD is much higher, with roughly $200-300$ cases per million people. In fact, for a long time after IBD was first reported in the United Kingdom in the mid-1800s, it was thought of as a disease of European ancestry. But with the condition now appearing all around the world, the consensus is that anyone can get IBD. "IBD really is a global disease now," says Ashwin Ananthakrishnan, an epidemiologist and clinician at the Massachusetts General Hospital Crohn's and Colitis Center in Boston. "If you look at the sheer number of patients, there are probably more in India and China than in North America."

The continuing surge in cases is providing research opportunities for clinicians such as Ng. Like other immune-related disorders such as multiple sclerosis and psoriasis, the incidence of IBD has risen in parallel with industrialization and urbanization, but the environmental drivers of IBD are not well understood. As people flock to cities, they have access to better health care and sanitation, but they are also exposed to poorer air quality, and are more 
likely to have sedentary, largely indoor lifestyles and to consume convenience diets that are high in saturated fat. Studies have linked factors such as these with IBD, but it is difficult to determine the origin in regions that have a long history of the disease. The ideal time to tease apart the complex web of environmental triggers and genetic associations, says $\mathrm{Ng}$, is as urbanization is happening and before the incidence of IBD has peaked. The extent of environmental change may correlate with IBD's rise and characteristics, and could lead to new hypotheses about the causes. "The golden time for finding the cause of the disease is the next ten years," says Ng.

\section{GENETIC CHALLENGE}

More than 200 genetic variants have been associated with IBD. In the first genetic-association study of IBD to include multiple ethnic groups - Europeans, East Asians, Indians and Iranians - 38 new loci (regions of the genome) with links to IBD were uncovered, 25 of which have previously been associated with other diseases or traits, such as multiple sclerosis or levels of cholesterol ${ }^{2}$.

But the rise of IBD in Asia is not being driven by the emergence of new mutations - genetic changes occur over a much longer timescale than the global onset of IBD has taken. That does not mean, however, that genetics has nothing to offer in the quest to understand the increase of incidence in Asia. Genetic variants could provide clues to why environmental changes have greater impacts on some populations than on others, says Ananthakrishnan. Punjab state in northern India, for instance, has one of the highest incidences of ulcerative colitis in Asia - around 6 new cases in 100,000 people per year ${ }^{3}$. And the incidence of IBD in people of Indian descent who live in Malaysia is six times higher than that in indigenous Malaysians, and three times higher than that in those of Chinese descent ${ }^{4}$; clearly, some genetic risk factor is at play.

Not all the genes or variants present the same risk across the globe. For example, the ATG16L1 gene - involved in the cellular recycling process autophagy - is associated with risk of Crohn's in white populations, but is not implicated in IBD in Asian people. And NOD2, a risk gene in individuals of European ancestry that is also connected with more aggressive forms of the disease, harbours different IBD-linked variants in Asian populations. What's more, the degree to which these genes explain the disease varies between different populations. In Asia, only 3\% of people with IBD have a close relative with the disease, compared with $15 \%$ of individuals in the West ${ }^{5}$.

As well as overall risk, certain gene variants may also correlate with particular presentations of IBD, Ananthakrishnan says. His work, which focuses on the environmental and genetic factors associated with IBD, suggests that an Indian person living in the West, for example, will have a type of Crohn's that is more similar

\section{DIAGNOSIS \\ Preparing for the burden}

Inflammatory bowel disease (IBD) poses a challenge to health-care systems everywhere. It's difficult to recognize, let alone treat, and therapies (biological drugs and surgery) are expensive and, in the case of medication, needed long-term, with costs often falling to the patient. In Asia, all indications are that "the prevalence and impact on the health-care system will continue to climb", says Siew Ng, a gastroenterologist at the Prince of Wales Hospital in Hong Kong. Jae Hee Cheon at Yonsei University College of Medicine, Seoul, thinks that "the incidence of IBD in East Asia will be at the same level to that of Western countries in the next 20 to 30 years".

Work to prepare countries to deal with the medical and economic burden of IBD is under way, but in Asia the starting point is especially low. Shortly after finishing her fellowship in London and starting work in Hong Kong, Ng realized the extent to which IBD was underdiagnosed, mainly because of lack of awareness. "There were patients who had the disease for a long time, but nobody told them it was probably IBD," she says.

The physicians who seem to be the most ready are often those who, like $\mathrm{Ng}$, trained in the West - where they were exposed to a significant number of people with IBD and then returned to Asia. For instance, on returning to Malaysia in 2003 after training in the United Kingdom, gastroenterologist Ida Hilmi at the University of Malaya in Kuala Lumpur was put in charge of diagnosing and treating IBD at her university's medical centre. Although mainly a clinician, she also set up an IBD registry to track new cases.
"Most of these patients are going to turn up in primary care. Doctors might have heard about IBD a long time ago in their medical school, but they haven't come across it. They don't think of the disease," Hilmi says. To try to change this, she has assembled an IBD special-interest group to promote awareness of the disease among health professionals and patients, and to distribute treatment algorithms to hospitals across Malaysia.

The technical challenge of diagnosing IBD should not, however, be underestimated. Many of the symptoms of IBD are common to other gastrointestinal conditions such as colorectal cancer, requiring physicians to rule out other potential diseases to reach a positive diagnosis. The process involves blood and stool tests, radiography and, most importantly, endoscopy. In Asia, diagnosis is further complicated by infectious diseases, including intestinal tuberculosis, which can present with near-identical symptoms to those of IBD. Physicians often start treatment for one or the other (the treatments are completely different) before patients get an accurate diagnosis. "We have many endemic infectious diseases. If someone has diarrhoea, they get multiple courses of antibiotics," Hilmi says.

In Korea, says Cheon, the complex nature of the diagnosis and treatment of IBD means that most people are treated at university hospitals. Because the availability of this level of care tends to increase with both wealth and urbanization, part of the concurrent rise in IBD in Asia may well be due to improved diagnosis, Ng says. K.R.C. to that seen in India than to cases in the West. Genetic and microbial data from different populations can also be used to help tailor treatments for people with IBD, says Jae Hee Cheon at Yonsei University College of Medicine in Seoul, who has conducted genetic, microbiome and epidemiological research in Koreans with IBD. For instance, the microbiota pills in development, which researchers hope to use to replace a pathogenic gut bacterial community with a healthy one, will not necessarily work for patients across the globe because patient populations vary in their disease features and response to treatment, he says.

And finding the relevant genetic variants in smaller Asian countries is difficult, partly because the disease is still relatively rare. In Malaysia, for example, only about 2,0003,000 people are thought to have IBD. That makes genome-wide association studies, which need many thousands of cases, nigh on impossible. The next best option for researchers is to pick handfuls of genetic variants identified as risk factors in larger studies, such as those done in the West, and test to see whether these variants pose a risk in smaller groups, says gastroenterologist Ida Hilmi at the University of Malaya in Kuala Lumpur.

\section{RISK FACTORS}

One of the biggest questions Ananthakrishnan fields from people with IBD is how they can prevent their children from developing the disease. "It's a question that all of us working in the environmental-influence space want to answer," he says. But at the moment, he can offer only a few general recommendations: avoid passive smoking, minimize antibiotic exposure in the early years and avoid giving children non-steroidal anti-inflammatory drugs, which can irritate the gut. For the most part, however, studies have shown these to be 


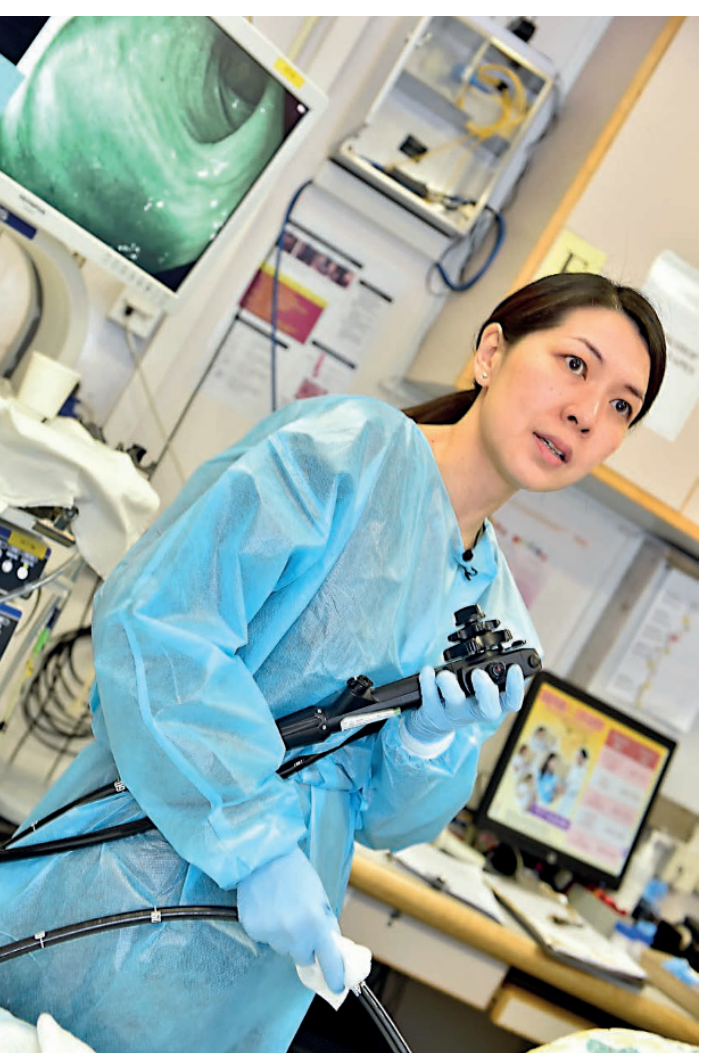

Siew Ng from the Prince of Wales Hospital in Hong Kong, performs a colonoscopy for IBD.

only risk factors, rather than proven interventions. Work to unpick the environmental factors behind IBD has a long way to go.

Some of the clearest evidence for the role of the environment in IBD comes from studies of immigrants. For example, a large study of residents in Ontario, Canada, showed that South Asian immigrants have an incidence rate of roughly 70 new cases of IBD per million people per year ${ }^{6}$. Although that is still considerably lower than the annual rate for long-term residents of 240 per million, children of South Asian immigrants have an annual incidence rate that is nearly the same ( 60 per million) as the children of long-term residents (72 per million). One of the biggest surprises in this study was that the age at which immigrants arrive in Canada is a strong predictive factor for developing IBD, says paediatric gastroenterologist Eric Benchimol of the Children's Hospital of Eastern Ontario Research Institute in Ottawa, who led the study. For every decade older the person is when they arrive, the risk of IBD decreases by nearly $10 \%$. This implies that there are factors in the new environment that, for certain genetic variations, increase the risk of IBD.

Researchers suspect that the likeliest way the environment increases risk is by altering a person's gut bacteria. The main line of thought is that environmental triggers have a greater influence early in life, as the immune system and a full complement of gut bacteria develop and mature. "That's where the excitement is," says

Benchimol. Several large studies are under way in the United States and Canada to investigate the theory. In 2015, researchers at the University of Pennsylvania in Philadelphia showed that inflammation, antibiotic exposure and changes to diet independently alter the balance of gut microbes in children with Crohn's disease ${ }^{7}$. Treatment with a biological drug to decrease inflammation or dietary approaches seemed to normalize the composition of the microbiota.

The ongoing Canadian Children Inflammatory Bowel Disease Network's CIDsCaNN study is collecting data from children with a new IBD diagnosis and following them for 18 months, including those with a South Asian background. Researchers are collecting stool samples to investigate the make-up of the microbiota, as well as recording information about early-life environmental risk factors, to try to understand what is triggering the disease. And a team at the University of Toronto

\section{"I can hardly keep up with all the patients Ihave, the number of cases is exploding."}

in Canada is running a study called GEMINI (Generational Differences in Environmental Exposures caused by Migration: Impact on Incidence of Inflammatory Disease), to investigate risk factors in healthy first- and secondgeneration South Asian immigrants for IBD and other autoimmune diseases (such as type 1 diabetes and multiple sclerosis). Canada is the perfect place to conduct these studies, partly because its national-health registries make it easy to identify and track those with the disease, Benchimol says.

\section{A CLOSING WINDOW}

Prospective studies such as CIDsCaNN, which follow individuals over time, are crucial for understanding the environmental factors that contribute to IBD. Ananthakrishnan is analysing data gathered through the Nurses' Health Study, a longitudinal study of more than 100,000 US women who have completed medical history and lifestyle questionnaires for decades. He found that women who ate high levels of fibre - about 24 grams per day - in the form of fruits and vegetables, were $40 \%$ less likely to get Crohn's disease than women who ate around half that ${ }^{8}$. Other studies have found similar trends for ulcerative colitis. High fibre intake has also been shown to prevent relapse in people with IBD, and animal models of IBD support the role of fibre in reducing the inflammatory damage wrought by the disease. Taken together, the move from a high-fibre diet towards a lowfibre Western diet, which seems to be happening in Asian cities in particular, might be part of the reason for the rise in IBD, Ananthakrishnan says.

One limitation to applying findings from Western studies, such as the Nurses' Health Study, to what is happening in Asia is that participants are mainly of European ancestry. Although some factors, such as breastfeeding, seem to be universally protective, other environmental factors do not always carry the same weight across different ethnicities.

Smoking, for instance, is one of the strongest risk factors for Crohn's disease in Western populations, but this was not the case for Asian participants in Ng's ACCESS cohort. ACCESS data also seem to suggest that antibiotic use - thought to be a risk factor in the West - is protective against IBD in Asian populations. But it can be more difficult to decipher relationships between antibiotic use and IBD in Asia, because the drugs can be bought over the counter in some countries, making it difficult to accurately record their use.

To get to the heart of why the incidence of IBD in Asia is on the rise, rigorous studies based in the continent are needed. Ananthakrishnan thinks that the data sets gathered in the West are excellent models for understanding how environmental risk factors affect chronic disease. Researchers can use these both to narrow the scope of the exposure data and determine when and how often data are collected in Asian epidemiological studies of IBD, says gastroenterologist and epidemiologist Gilaad Kaplan of the University of Calgary in Canada.

$\mathrm{Ng}$ and her collaborators are pursuing several lines of research as the incidence in Asia swells and their golden window of opportunity to identify the triggers begins to diminish. She is planning to compare the urban and rural populations of China, which are ethnically similar, but starkly different in their environmental exposures - from their dietary choices to whether they use flush toilets. Targeting areas in China that have a low incidence of IBD, such as Xiangshan County in Zhejiang and Chengdu, may help to uncover genetic and environmental factors that protect some individuals from the disease. Much research needs to be done, $\mathrm{Ng}$ says. "Every day, I go to work and I see new cases being referred, and I ask my colleague, 'Why has this disease dropped from the sky, out of nowhere? Where did it come from?" Researchers know that the best chance to find out is now.

Kelly Rae Chi is a freelance science writer in Cary, North Carolina

1. Ng, S. C. et al. Inflamm. Bowel Dis. 22, 1954-1960 (2016).

2. Liu, J. Z. et al. Nature Genet. 47, 979-986 (2015).

3. Sood, A. et al. Gut 52, 1587-1590 (2003).

4. Hilmi, I., Jaya, F., Chua, A., Heng, W. C., Singh, H., Goh, K. L. J. Crohn's Colitis 9, 404-409 (2015).

5. Ng, S. C. Gastroenterol. Hepatol. 12, 193-196 (2016).

6. Benchimol, E. I. et al. Am. J. Gastroenterol. 110, 553-563 (2015).

7. Lewis, J. D. et al. Cell Host Microbe 18, 489-500 (2015).

8. Ananthakrishnan, A. N. et al. Gastroenterology 145, 970-977 (2013). 